\title{
LOWER BOUNDS FOR THE EXTRINSIC TOTAL CURVATURES OF A SPACE-LIKE CODIMENSION 2 SURFACE IN MINKOWSKI SPACE
}

\author{
MAREK KOSSOWSKI \\ (Communicated by Jonathan M. Rosenberg)
}

\begin{abstract}
There are three invariant curvature functions defined on any smooth space-like 2-surfaces in four-dimensional Minkowski space. (If the surface lies in a Euclidean hyperplane then the functions agree with $H^{2}, K^{2}$, and $\left(H^{2}-K\right)^{2}$. For each of these functions we show that there exists a space-like immersion of any oriented compact (or noncompact complete) surface with associated total curvature arbitrarily small.
\end{abstract}

A two dimensional oriented surface $M$ in four dimensional Minkowski space which inherits a positive definite metric is called space-like. Such a surface carries three functions $\mathbf{H}, \mathbf{K}$, and $\mathbf{U}$ that are determined by the first and second fundamental forms. (If the surface lies in a Euclidean hyperplane then these functions reduce to $H^{2}, K^{2}$, and $\left(H^{2}-K^{2}\right)$ respectively.) It is natural to ask for the infimum of the respective total curvatures $\int_{M}|\mathbf{H}| d A, \int_{M}|\mathbf{K}|^{1 / 2} d A$ and $\int_{M} \mathbf{U}^{1 / 2} d A$ over the set of embedded compact space-like surfaces. In this paper we show that there exists an embedded smooth compact orientable surface $M_{g}$ of genus $g \geq 0$ with $\int_{M g}|\mathbf{H}| d A$ arbitrarily small (Theorem 5). In the process we relate $\mathbf{H}$ to the split mean curvatures in the manner of reference [2]. We then show that there exist compact (and complete noncompact) embedded orientable surfaces of arbitrary genus with either $\mathbf{K}$ or $\mathbf{U}$ identically zero, (Theorems 6 and 7). The author would like to thank P. Ehrlich, R. Howard, T. K. Milnor and R. Penrose for inspiration.

Throughout this paper $\mathbf{M}^{4}$ will denote Minkowski space, the real four dimensional vector space equipped with bilinear form $\langle$,$\rangle of type (3,1)$, (i.e., the normal form has 3 plus signs and 1 minus sign). We will assume that $\mathbf{M}^{4}$ is oriented and time oriented (i.e., a 4-volume form $d V$, and future time-like vector field Fut, have been chosen.) We will write $L C=\left\{v \in \mathbf{M}^{4} \mid\langle v, v\rangle=0\right\}$ to denote the light cone in Minkowski space. A space-like surface in $\mathbf{M}^{4}$ is

Received by the editors February 24, 1989 and, in revised form, September 11, 1989.

1980 Mathematics Subject Classification (1985 Revision). Primary 53C50; Secondary 53B30, $53 \mathrm{C} 42$.

Research partially supported by NSF grant DMS 88-03585. 
a smooth immersion of a smooth compact oriented 2-manifold $k: M \rightarrow \mathbf{M}^{4}$, such that the induced metric $k^{*}\langle$,$\rangle is positive definite. It follows that the$ normal bundle $M^{\perp} \rightarrow M$ is a trivial rank two bundle with fibres carrying a type $(1,1)$ metric. Now choose an orthogonal splitting of Minkowski space $\mathbf{M}^{4}=\mathbf{E}^{3}+\mathbf{E}^{-}$with projections $t: \mathbf{E}^{3}+\mathbf{E}^{-} \rightarrow \mathbf{E}^{-}$and $\pi_{\mathbf{E}}: \mathbf{E}^{3}+\mathbf{E}^{-} \rightarrow \mathbf{E}^{3}$ and let $U_{s}^{i}, i=F, P$ denote the two unique sections of $M^{\perp}$ which satisfy the following:

(a) $\left\langle U_{s}^{i}, U_{s}^{i}\right\rangle=0, \quad i=F, P$,

(b) $t_{*}\left(U_{s}^{i}(x)\right)$ is of unit length for all $x \in M, \quad i=F, P$,

(c) $\left\langle U_{s}^{F}\right.$, FUT $\rangle<0$,

(d) $d V\left(-,-, U_{s}^{p}, U_{s}^{F}\right) \equiv d A(-,-)$,

(Here $d A$ is the induced area form on $M$ and equivalence is in the sense of orientations. The subscript $s$ refers to the choice of splitting.)

We also have the splitting dependent function $\frac{1}{\sqrt{2}}\left\langle U_{s}^{F}, U_{s}^{P}\right\rangle: M \rightarrow R$, which we denote by TILT $_{s}$, and which satisfies:

(a) $0<\operatorname{TILT}_{s} \leq 1$

(b) TILT $_{s}$ is constant iff $\operatorname{TILT}_{s}=1$ and $M$ lies in a fiber of $t: \mathbf{E}^{3}+\mathbf{E}^{-} \rightarrow \mathbf{E}^{-}$.

See Lemma 2 in [1].

Now for any choice of splitting $\mathbf{M}^{4}=\mathbf{E}^{3}+\mathbf{E}^{-}$we get two split second fundamental forms $\Pi_{s}^{i}: T M \times T M \rightarrow \mathbf{R}, i=F, P$ defined by $\Pi_{s}^{i}(X, Y)=$ $\left\langle\nabla_{X} U_{s}^{i}, Y\right\rangle$ where $X, Y$ are vector fields on $M$ and $\nabla$ is the ambient connection. We define the split curvatures by $K_{s}^{i}=\frac{\mathrm{DET} \Pi_{s}^{i}}{\mathrm{DETI}}, i=F, P$ and split mean curvatures by $H_{s}^{i}=\frac{1}{2} \frac{\mathrm{TR} \Pi_{s}^{i}}{\mathrm{DETI}}, i=F, P$ where $I$ is the first fundamental form on $M$.

For any normal vector $u_{x} \in M_{x}$, we have an endomorphism of $T_{x} M$ defined by $v_{x} \rightarrow$ (tangential projection of $\nabla_{v} u$ at $x$ ). There are unique global sections $R^{F}, R^{P}$ of $M$ that satisfy $\left\langle R^{F}, R^{P}\right\rangle=\sqrt{2}$ and a,c, and $\mathrm{d}$ of (E1) above. For such a pair we have the quadric mean curvature $\mathbf{H}=\frac{1}{4} \operatorname{TR}\left(\nabla R^{F}\right) \cdot \operatorname{TR}\left(\nabla R^{P}\right)$ and the quartic curvature $\mathbf{K}=\operatorname{DET}\left(\nabla R^{F}\right) \cdot \operatorname{DET}\left(\nabla R^{P}\right)$. Observe that if we fix a splitting $\mathbf{M}^{4}=\mathbf{E}^{3}+\mathbf{E}^{-}$, then

$$
\begin{aligned}
\mathbf{H} & =\frac{H_{s}^{F} H_{s}^{P}}{\mathrm{TILT}_{s}}, \text { and } \\
\mathbf{K} & =\frac{K_{s}^{F} K_{s}^{P}}{\left(\mathrm{TILT}_{s}\right)^{2}}
\end{aligned}
$$

From these splitting dependent descriptions of $\mathbf{H}$ and $\mathbf{K}$ it is apparent that

$$
\mathbf{U}=\frac{\left(K_{s}^{F}-\left(H_{s}^{F}\right)^{2}\right)\left(K_{s}^{P}-\left(H_{s}^{P}\right)^{2}\right)}{\left(\mathrm{TILT}_{s}\right)^{2}} \geq 0
$$


is also an invariant, which we will call the umbilic curvature. All three functions $\mathbf{H}, \mathbf{K}, \mathbf{U}: M \rightarrow \mathbf{R}$ are congruence invariants for $(M, k)$.

Proposition 1. Given $(M, k)$, then for all splittings $\mathbf{M}^{4}=\mathbf{E}^{3}+\mathbf{E}^{-}$,

$$
\int_{M}|\mathbf{H}| d A \leq\left[\sup \frac{1}{2 \mathrm{TILT}_{s}}\right] \int_{M}\left(H_{s}^{F}\right)^{2}+\left(H_{s}^{P}\right)^{2} d A .
$$

Further if equality holds for some splitting then $(M, k)$ is an immersion into a space-like hyperplane $\mathbf{E}^{3} \subset \mathbf{M}^{4}$. (The supremum is taken over all splittings $\mathbf{M}^{4}=\mathbf{E}^{3}+\mathbf{E}^{-}$).

Proof. Choose a splitting. Then (E3) implies that

$$
2|\mathbf{H}| \leq \frac{1}{\operatorname{TILT}_{s}}\left[\left(H_{s}^{F}\right)^{2}+\left(H_{s}^{P}\right)^{2}\right]
$$

from which follows the inequality

$$
2 \int_{M}|\mathbf{H}| d A \leq \int_{M} \frac{\left(H_{s}^{F}\right)^{2}+\left(H_{s}^{P}\right)^{2}}{\operatorname{TILT}_{s}} d A \leq\left[\sup \frac{1}{\operatorname{TILT}_{s}}\right] \int_{M}\left(H_{s}^{F}\right)^{2}+\left(H_{s}^{P}\right)^{2} d A .
$$

Since equality implies $\left(\mathrm{TILT}_{s}\right)$ is constant (E2b) implies our claim.

Theorem 2. Given $(M, k)$ then for all splittings $\mathbf{M}^{4}=\mathbf{E}^{3}+\mathbf{E}^{-}$,

$$
8 \pi \leq\left[\sup \frac{1}{\operatorname{TILT}_{s}}\right] \int_{M}\left(H_{s}^{F}\right)^{2}+\left(H_{s}^{P}\right)^{2} d A .
$$

Further if equality holds for some splitting then $M=S^{2}$ and $k$ embeds $S^{2}$ as a round sphere in a space-like hyperplane $\mathbf{E}^{3} \subset \mathbf{M}^{4}$.

Proof. Recall that for any splitting we have:

$$
K_{s}^{i} \leq\left(H_{s}^{i}\right)^{2} \quad i=F, P .
$$

with equality at $x \in M$ if and only if $x$ is an umbilic point for $\Pi_{s}^{i}, i=F, P$. Now with (E7) of [1] we have that

$$
\begin{aligned}
8 \pi & \leq \int_{\left\{K_{s}^{F}>0\right\}} K_{s}^{F} d A+\int_{\left\{K_{s}^{P}>0\right\}} K_{s}^{P} d A \\
& \leq \int_{\left\{K_{s}^{F}>0\right\}}\left(H_{s}^{F}\right)^{2} d A+\int_{\left\{K_{s}^{P}>0\right\}}\left(H_{s}^{P}\right)^{2} d A \\
& \leq \int_{M}\left(H_{s}^{F}\right)^{2}+\left(H_{s}^{P}\right)^{2} d A \\
& \leq\left[\sup \frac{1}{\operatorname{TILT}_{s}}\right] \int_{M}\left(H_{s}^{F}\right)^{2}+\left(H_{s}^{P}\right)^{2} d A
\end{aligned}
$$

Finally if we have equality, then $\operatorname{TILT}_{s}=1$, hence $(M, k)$ is an immersion into an $\mathbf{E}^{3}$ factor of the splitting with $\mathbf{E}^{3}$-mean curvature given by $H_{s}^{F}=H_{s}^{P}$ and Gaussian curvature given by $K_{s}^{F}=K_{s}^{P}$. Thus $(M, k)$ is a totally umbilic surface in a $\mathbf{E}^{3} \subset \mathbf{M}^{4}$. 
Next we examine how the split mean curvatures behave individually.

\section{Theorem 3.}

(a) Given $(M, k)$ with $M=S^{2}$, then for all splittings $\mathbf{M}^{4}=\mathbf{E}^{3}+\mathbf{E}^{-}$

$$
4 \pi \leq \int_{S^{2}}\left(H_{s}^{i}\right)^{2} d A \quad i=F, P
$$

Further equality for $i=F$ or $P$ implies that $k$ embeds $S^{2}$ in a light cone $L C \subset \mathbf{M}^{4}$. Equality in both $i=F$ and $P$ implies that $(M, k)$ is congruent to a round $S^{2}$ in a space-like hypersurface $\mathbf{E}^{3} \subset \mathbf{M}^{4}$.

(b) Given $\varepsilon>0$ and an integer $g>0$, then there exists an orientable compact space-like embedded surface of genus $g$ and a splitting $\mathbf{M}^{5}=\mathbf{E}^{3}+\mathbf{E}^{-}$such that for $i=F$ or $P$

$$
0 \leq \int_{M_{g}}\left(H_{s}^{i}\right)^{2} d A \leq \varepsilon
$$

(Note that because of (E5) above this holds for only one of the split mean curvatures.)

Proof. (a) Referring to Theorem 1 of [1] we have that

$$
4 \pi \leq \int_{\left\{K_{s}^{i}>0\right\}} K_{s}^{i} d A, \quad i=F, P
$$

where the integration is over the subset of $M$ where $K_{s}^{i}>0$. As a consequence we have that for $i=F, P$,

$$
\begin{aligned}
4 \pi & \leq \int_{\left\{K_{s}^{i}>0\right\}} K_{s}^{i} d A \\
& \leq \int_{\left\{K_{s}^{i}>0\right\}}\left(H_{s}^{i}\right)^{2} d A \leq \int_{s^{2}}\left(H_{s}^{i}\right)^{2} d A .
\end{aligned}
$$

Now suppose there is equality for $i=F$ or $P$. From (E6) of [2] it follows that as conformal structures on $M, \Pi_{s}^{i}$ for $(M, k)$ agrees with $\bar{\Pi}$ for the light-like hypersurface $\operatorname{LL}^{i}(M, k)$. Thus if we choose any (affine) spacelike hyperplane $\mathbf{E}^{3} \subset \mathbf{M}^{4}$, at any immersed point in $\left\{\mathbf{E}^{3} \cap \operatorname{LL}^{i}(M, k)\right\}$ this intersection is locally an umbilic surface in $\mathbf{E}^{3}$, and hence is a piece of a round sphere or of a plane. Since we are assuming $M$ is compact, we will have our result if we show that there exists an $\mathbf{E}^{3} \subset \mathbf{M}^{4}$ with intersection $\left\{\mathbf{E}^{3} \cap \mathbf{L L}^{i}(M, k)\right\}$ consisting of only immersive points (i.e., $\operatorname{LL}^{i}(M, k)$ is an icon). To prove this choose a hyperplane $\widetilde{\mathbf{E}}^{3} \subset \mathbf{M}^{4}$ so that the intersection $\left\{\widetilde{\mathbf{E}}^{3} \cap \mathbf{L L}{ }^{i}(M, k)\right\}$ contains an immersive point. Recall that $\operatorname{LL}^{i}(M, k) \stackrel{\pi^{i}}{\longrightarrow} M$ is fibered by null lines and each fiber intersects the chosen $\widetilde{\mathbf{E}}^{3}$ in a unique point. (See [1] for details.) Since $k: M \rightarrow \mathbf{M}^{4}$ has image, which is a section of this fibering of $\operatorname{LL}^{i}(M, k)$, 
we now have smooth maps

$$
\begin{aligned}
S^{2}=M \stackrel{k}{\rightarrow} & \operatorname{LL}^{i}(M, k) \subset \mathbf{M}^{4} \\
& \downarrow p \\
& \widetilde{\mathbf{E}}^{3} \subset \mathbf{M}^{4}
\end{aligned}
$$

where $p \circ k(x)$ is intersection point of the $\pi^{i}$-fibered through $k(x)$ and the chosen $\widetilde{\mathbf{E}}^{3}$. We know the immersive points of $p \circ k$ are open in $S^{2}=M$, we need only show that they are closed. Let $x_{n} \rightarrow x$ be a convergent sequence of points in $S^{2}$ with $p \circ k$ immersive at $x_{n}$. Now consider the $\mathbf{E}^{3}$ parallel to the above chosen $\widetilde{\mathbf{E}}^{3}$ that contains $k(x)$. We know that $\left\{\mathbf{E}^{3} \cap \operatorname{LL}^{i}(M, k)\right\}=V$ is a piece of a sphere or a plane. Hence $\left(\pi^{i}\right)^{-1}\left(\pi^{i}(V)\right)$ is a subset of a light cone or a null 3-plane. But for any affine $\mathbf{E}^{3} \subset \mathbf{M}^{4}$, the intersection of $\mathbf{E}^{3}$ with a light cone or a null 3-plane consists entirely of immersive points or entirely of nonimmersive points. Thus the intersection of $\left(\pi^{i}\right)^{-1}\left(\pi^{i}(V)\right) \subset \operatorname{LL}^{i}(M, k)$ with the chosen $\widetilde{\mathbf{E}}^{3}$ contains $p \circ k\left(x_{n}\right)$ (for $n$ sufficiently large). These are immersive points for $p \circ k$ and hence immersive points for the intersection with $\widetilde{\mathbf{E}}^{3}$. Since $p \circ k(x)$ lies in this intersection it is also an immersive point. We conclude $\operatorname{LL}^{i}(M, k)$ is congruent to a light cone. For the last claim in Theorem 3 (a) we now know that both $\operatorname{LL}^{i}(M, k)$ are congruent to light cones. Hence $(M, k)$ is space-like, compact, and lies in their intersection. It must be a round $S^{2} \subset \mathbf{E}^{3} \subset \mathbf{M}^{4}$.

(b) The construction of $\left(M_{g}, k\right)$ is but a slight modification of the construction presented in Appendix 2 of [1]. Consider a fixed splitting $\mathbf{M}^{4}=\mathbf{E}^{3}+\mathbf{E}^{-}$ with projection $t: \mathbf{E}^{3}+\mathbf{E}^{-} \rightarrow \mathbf{E}^{-}$and the torus of revolution $\left(T^{2}, k\right)$ obtained by rotating an "embedded Figure 8 " about a $\mathbf{M}^{2}$ in $\mathbf{M}^{4}$ so tha a linear segment in the figure 8 generates a cylinder in $\mathbf{E}^{3}$; and the $S^{2}$ valued Gauss maps are not surjective. For this torus and splitting $H_{s}^{i}=\frac{1}{2} \frac{\operatorname{TR}\left\langle\nabla U_{s}^{i},\right\rangle}{\mathrm{DETI}}$, $i=F, P$. Now because the Gauss maps are not surjective, there exist isometries $b_{\theta}: \mathbf{M}^{4} \rightarrow \mathbf{M}^{4}$ such that for all positive integers $n>0$, there exist $\theta_{n}$ so that $n<\operatorname{INF}\left|t_{*}\left(b_{\theta_{n}} \circ U_{s}^{F}\right)\right|$ where the infimum is over points of the torus. Since $b_{\theta_{n}}$ is an isometry we have that $\left(T^{2}, b_{\theta_{n}} \circ k\right)$ has $U_{s \theta_{n}}^{F}=\lambda\left(b_{n} \circ U_{s}^{F}\right)$ where $\lambda>\frac{1}{n}$ is a function on $T^{2}$ and $U_{s \theta_{n}}^{F}$ is the vector field satisfying (E1) above relative to the fixed splitting. We have that,

$$
H_{s \theta_{n}}^{F}=\frac{\operatorname{TR}\left\langle\nabla U_{s \theta_{n}}^{F},-\right\rangle}{\text { DET I }}<\frac{1}{n} \frac{\operatorname{TR}\left\langle\nabla U_{s}^{F},-\right\rangle}{\text { DET I }}<\frac{H_{s}^{F}}{n}
$$

and hence a torus with $\int_{T^{2}}\left(H_{s}^{F}\right)^{2} d A$ arbitrarily small. Note that the key step of constructing $b_{\theta_{n}}$ requires only that the Gauss map be nonsurjective. Thus to construct higher genus examples take two copies of $\left(T^{2}, k\right)$ and shrink one 
copy so that the two copies do not intersect, and the cylinders in $\mathbf{E}^{3}$ mentioned at the outset are concentric. We can connect these two cylinders in the $\mathbf{E}^{3}$ with catanoidal necks in such a way that removing the $\mathbf{E}^{3}$-minimal parts of the necks results in two punctured tori with $\mathbf{M}^{4}$-Gauss maps nonsurjective. If we denote this connected sum by $\left(M_{g}, k\right)$ then for any splitting $\mathbf{M}^{4}=\mathbf{E}^{3}+\mathbf{E}^{-}$, both the $H_{s}^{i}, i=F, P$ will vanish on the $\mathbf{E}^{3}$-minimal part of the necks. Thus we may repeat the above argument on $\left(M_{g}-\left\{H_{s}^{F}=0\right\}, b_{\theta_{n}} \circ k\right)$ and we have surfaces which satisfy our claim for $i=F$. The same argument adapts to construct an example for $i=P$.

By a space-like graph in $\mathbf{M}^{4}$ we mean a smooth space-like hypersurface which, relative to some (hence any) splitting of $\mathbf{M}^{4}=\mathbf{E}^{3}+\mathbf{E}^{-}$is globally the graph of a function $f: \mathbf{E}^{3} \rightarrow \mathbf{E}^{-}$.

Theorem 4. If $(M, k)$ is an embedding into a space-like graph, then for all splittings of $\mathbf{M}^{4}=\mathbf{E}^{3}+\mathbf{E}^{-}$

$$
4 \pi \leq \int_{M}\left(H_{s}^{i}\right)^{2} d A, \quad i=F, P .
$$

Further equality for $i=F$ or $P$ implies that $M=S^{2}$ so that Theorem 3, Part (a) applies.

Proof. Recall from the proof of Theorem 9 in [1] that for $(M, k)$ a surface embedded in a graph we must have

$$
4 \pi \leq \int_{\left\{K_{s}^{i}>0\right\}} K_{s}^{i} d A, \quad i=F, P .
$$

(This is a restatement of the first line in the proof of Theorem 9.) Now for the case of equality we must have by Theorem 1 of [1] that $M=S^{2}$.

Theorem 5. Given $\varepsilon>0$ and an integer $g \geq 0$, there exists an orientable compact embedded space-like surface of genus $g$ with,

$$
0<\int_{M_{g}}|\mathbf{H}| d A<\varepsilon
$$

Proof. We first construct an example with $g=0$. Take two round spheres $S_{1}^{2}$ and $S_{2}^{2}$ in $\mathbf{E}^{3} \subset \mathbf{M}^{4}$ and smoothly connect them with a "Hopf neck" $H N \subset \mathbf{E}^{3}$ of small total squared $\mathbf{E}^{3}$-mean curvature to yield an embedded sphere, $S^{2} \# S^{2}$. This can be done as in Figure 1 so that the transition region between round sphere and Hopf neck consist of a pair of planar parallel anuli in $\mathbf{E}^{3}$. (For details concerning the Hopf neck see Lemma $6 \mathrm{~b}$ in [3].) Let $\mathbf{P}_{1}$ and $\mathbf{P}_{2}$ be the parallel planes in which these annuli lie. Now extend the $S^{2} \# S^{2} \subset \mathbf{E}^{3}$ to a Light-like hypersurface $\operatorname{LL}\left(S^{2} \# S^{2}\right)$ in $\mathbf{M}^{4}$ so that the vertex of round spherical parts lie in the "future" of the $\mathbf{E}^{3}$ fixed at the outset. Similarly extend the round spheres $S_{1}^{2}$ and $S_{2}^{2}$ to light cones $\mathrm{LC}_{1}$ and $\mathrm{LC}_{2}$ so that their vertices are in the future of the $\mathbf{E}^{3}$. Next extend the 2-planes $\mathbf{P}_{1}$ and $\mathbf{P}_{2}$ to null 3-planes $\mathbf{N}_{1}^{3}$ 


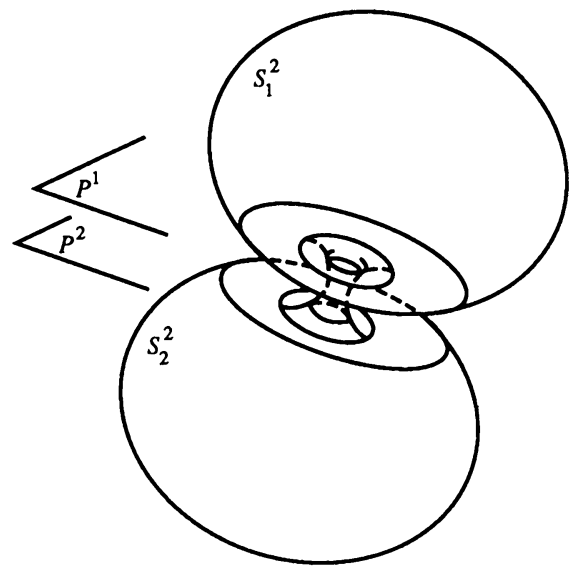

Figure 1

and $\mathbf{N}_{2}^{3}$ so that $\mathbf{N}_{\alpha}^{3}$ intersects the future of $\mathrm{LC}_{\alpha}$ in a bounded set. Let $\overline{\mathbf{N}}_{\alpha}^{3 F}$, $\alpha=1,2$ denote the closure of the subsets of $\mathbf{N}_{\alpha}^{3}$ that lie in the future of $\mathbf{E}^{3}$ and let $D_{\alpha}=\operatorname{LL}\left(S^{2} \# S^{2}\right) \cap \overline{\mathbf{N}}_{\alpha}^{3 F}, \alpha=1,2$. These $D_{\alpha}$ are embedded spacelike 2-disks. Thus by construction $\left\{D_{1} \cup H N \cup D_{2}\right\}$ is an embedded space-like two-sphere that intersects the fixed $\mathbf{E}^{3}$ in the Hopf neck along with its pair of planar annuli. Now since each $D_{\alpha}$ lies in a null 3-plane $\mathbf{H}$ vanishes on both $D_{\alpha}$. Thus the support of $\mathbf{H}$ lies entirely in the Hopf neck where it agrees with the $\mathbf{E}^{3}$-mean curvature squared. We have our sphere. To construct higher genus examples we need only connect the parallel annuli with additional Hopf necks with small total squared $\mathbf{E}^{3}$-mean curvature and we are finished.

We now construct space-like surfaces with $\mathbf{K}$ and $\mathbf{U}$ identically zero. We remark that the existence of $\mathbf{K}$-flat surfaces is relevant to the conjecture discussed in Appendix 3 of [1] in that these surfaces indicate that there need not exist a point $x \in M$ where both split curvatures are positive $K_{s}^{i}(x)>0 \quad i=F, P$.

Theorem 6. Given $g \geq 0$, there exist orientable embedded compact (or noncompact complete) space-like surfaces of genus $g$ with $\mathbf{K}=0$.

Proof. Consider any two $\mathbf{E}^{3}$-flat surfaces $\bar{k}^{i}: \mathrm{FLT}^{i} \rightarrow \mathbf{E}^{3} \subset \mathbf{M}^{4}, i=F, P$ that intersect transversely on an immersed circle $\tilde{S}^{1} \rightarrow \mathrm{FLT}^{F} \cap \mathrm{FLT}^{P}$. (The reason for indexing these surfaces by $F$ and $P$ will be apparent below.) Next choose $\mathbf{E}^{3}$-normals for each surface $n^{i}: \mathrm{FLT}^{i} \rightarrow S^{2} \subset \mathbf{E}^{3}, i=F, P$. As in the proof of Proposition 6 of [2] we use these $\mathbf{E}^{3}$-normals to construct two light-like hypersurfaces $j^{i}: \mathrm{LL}^{i}\left(\mathrm{FLT}^{i}\right) \rightarrow \mathbf{M}^{4}, i=F, P$. Now two null 3-planes in $\mathbf{M}^{4}$ intersect in an $\mathbf{E}^{2} \subset \mathbf{M}^{4}$ or coincide. Since $\mathrm{FLT}^{i}$ intersect transversely in $\mathbf{E}^{3}$ we have that $\operatorname{LL}^{F}\left(\mathrm{FLT}^{F}\right)$ intersects $\operatorname{LL}^{P}\left(\mathrm{FLT}^{P}\right)$ transversely in $\mathbf{M}^{4}$. Thus near 
the intersecting circle we have that $\mathrm{LL}^{F}\left(\mathrm{FLT}^{F}\right) \cap \mathrm{LL}^{P}\left(\mathrm{FLT}^{P}\right)$ is an immersed space-like cylinder $\widetilde{S}^{1} \times(-\delta, \delta)$. (It will be embedded if the $\mathrm{FLT}^{i} \subset \mathbf{E}^{3}$, $i=F, P$ are embedded.) It follows that this small cylinder is transverse to the fibers of both $\pi^{i}: \operatorname{LL}^{i}\left(\mathrm{FLT}^{i}\right) \rightarrow \mathrm{FLT}^{i}$. We may view the original $\mathrm{FLT}^{i}$ $i=F, P$ as "zero sections" of $\pi^{i}$ and observe that the intersection cylinder is transverse to both of these zero sections in $\operatorname{LL}^{i}\left(\mathrm{FLT}^{i}\right)$. Thus we may extend the $\widetilde{S}^{1} \times(-\delta, \delta)$ so that one boundary edge $\widetilde{S}^{1} \times\{-\delta\}$ extends so as to agree with the zero section of $\pi^{F}$ outside of a compact set and the other boundary edge $\widetilde{S}^{1} \times\{\delta\}$ extends so as to agree with the zero section of $\pi^{P}$ outside a compact set. We may construct this extension so that it is everywhere transverse to (at least) one of the fibrations $\pi^{i}, i=F, P$, thence this extended intersection $\left(\mathrm{FLT}^{F} \# \mathrm{FLT}^{P}, k\right)$ is a space-like surface in $\mathbf{M}^{4}$.

In Figure 3 we illustrate our construction and include the image of the extended intersection under the projection $\pi_{\mathbf{E}}: \mathbf{M}^{4} \rightarrow \mathbf{E}^{3}$. Now (E10) of [2] tells us that any section of either $\operatorname{LL}^{i}\left(\mathrm{FLT}^{i}\right) i=F, P$ must have $\mathbf{K}$ identically zero. Using the construction on flat surfaces as represented by Figures 2 and 3 we can build spheres or tori and take connected sums so that the resulting surfaces have $\mathbf{K}$ identically zero. We have our surfaces.

Theorem 7. Given $g \geq 0$ there exists an orientable embedded compact (or noncompact complete) space-like surface of genus $g$ with $\mathbf{U}=0$.

Proof. The proof parallels that of Theorem 6 with the modification that flat surfaces in $\mathbf{E}^{3}$ are replaced by round spheres $S_{i}^{2}, i=F, P$ in $\mathbf{E}^{3}$. The key point is again (E10) of [2], which in this context tells us that any section of either $\operatorname{LL}^{i}\left(S_{i}^{2}\right) \quad i=F, P$ must have $\mathbf{U}$ identically zero.

It should be apparent to the reader that these subumbilic immersions are highly nonrigid. So that $\mathbf{F}(M, k)=\int_{M} U^{1 / 2} d A$ defines a geometric functional

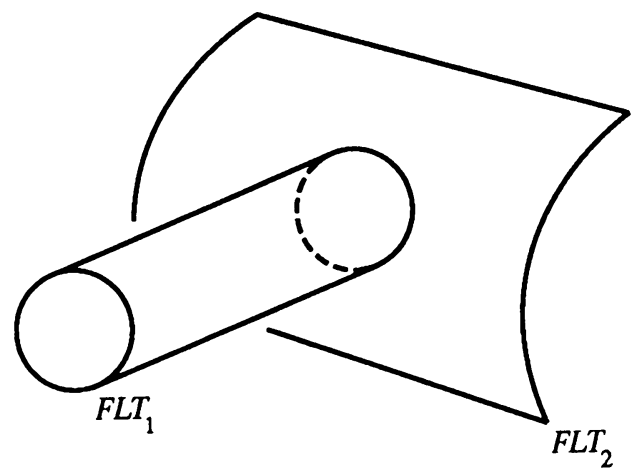

FIGURE 2 


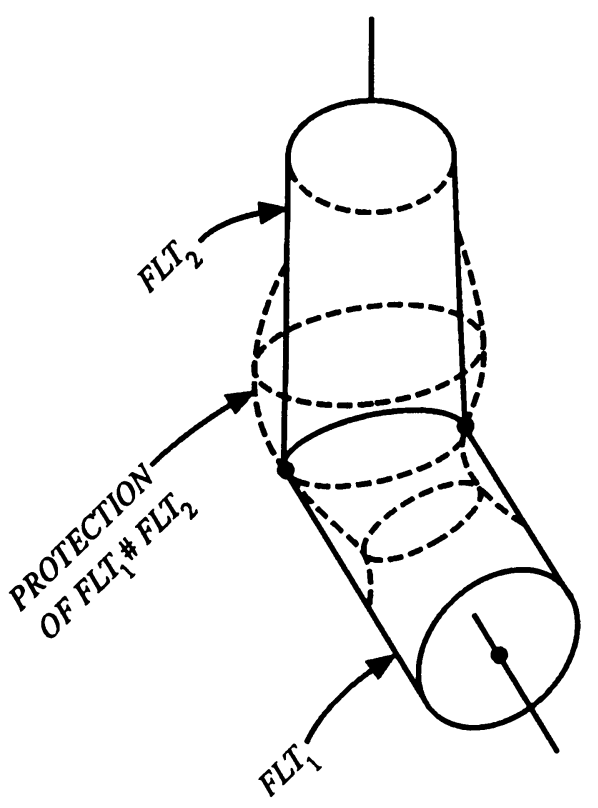

FIGURE 3

on $\operatorname{Immer}^{\infty}\left(M, \mathbf{M}^{4}\right)$ with highly degenerate second variation at the minima constructed above.

\section{BIBLIOGRAPHY}

1. J. K. Beem and P. E. Ehrlich, Global Lorentzian, Geometry, Dekker, 1981.

2. M. Kossowski, The $S^{2}$ valued Gauss maps and split total curvature of a space-like codimension 2 surface in Minkowski space, J. London Math. Soc. 40.

3. _ The intrinsic conformal structure and Gauss map of a Light-like hypersurface in Minkowski space, Trans. Amer. Math. Soc. 316 (1989).

4. W. Kuhnel and U. Pinkall, On total mean curvatures, Quart. J. Math. Oxford 37 (1986).

5. T. K. Milnor, Harmonic maps and classical surface theory in Minkowski 3-space, Trans. Amer. Math. Soc. 280 (1983).

6. B. O'Neil, Semi-Riemannian geometry with applications to relativity, Academic Press, New York, 1983.

7. R. Penrose, Battille Recontares, Benjamin, NY, 1968.

8. T. J. Wilmore, Total curvature in Riemannian geometry, Wiley, 1982.

Department of Mathematics, University of South Carolina, Columbia, South CarOLINA 29208 